\title{
El Trabajo Social relacional: una perspectiva para rastrear la configuración socioambiental del riesgo de inundación en los asentamientos precarios
}

\author{
Amanda Patricia Amorocho Pérez ${ }^{1}$; Dolly Cristina Palacio Tamayo²
}

Recibido: 16/06/2017 / Revisado: 27/09/2017 / Aceptado: 14/12/2017

Resumen. En la actualidad, las implicaciones y las consecuencias de las interacciones humanas en la sostenibilidad socioambiental y ecológica y en la construcción del riesgo son incontestables. Desde esta perspectiva, el Trabajo Social hoy tiene una oportunidad para recuperar las bases de su teoría social sobre la relación entre "la persona y su entorno" y aplicarlas a las interacciones socioambientales de las comunidades vulnerables, relativizando su papel en la configuración de los desastres por inundación en los asentamientos precarios. En este sentido, en este artículo presentamos una perspectiva relacional que indaga en las interacciones socioambientales de las poblaciones que migran por desplazamiento forzado, aplicando el análisis de redes sociales (ARS), para comprender la configuración socioambiental del riesgo de inundación en los asentamientos precarios, con la ilustración de un caso en Colombia. Nos encontramos con redes de migrantes que fundan asentamientos precarios en zonas de protección ambiental con una precaria percepción del riesgo - cuando hay desesperación, falta de oportunidades y de acceso a la tierra- para la construcción de viviendas, que conforma unos vínculos institucionales informales que sostienen el desarrollo del asentamiento con favores políticos. Reconocer la formación de estas redes es una oportunidad para reflexionar acerca de sus formas de pensar y de actuar, así como estas son una pieza importante de información para proponer escenarios de diálogo entre los actores implicados que, a su vez, facilite la definición de acuerdos colectivos que construyan ambientes más seguros y sostenibles.

Palabras clave: Migración; redes socioambientales del lugar; análisis de redes sociales; configuración socioambiental del riesgo de inundación; asentamientos precarios

\section{[en] Relational Social Work: a perspective to trace the socioenvironmental shaping of flood risk in precarious settlements}

\begin{abstract}
The implications and consequences of human interactions on socioenvironmental and ecological sustainability and on the construction of risk are now indisputable. From this perspective, contemporary social work has an opportunity to recover the bases of its social theory regarding the relationships between "person and environment", applying them to the socio-environmental interactions of vulnerable communities, qualifying their role in the shaping of disasters caused by flooding in precarious settlements. In this article, we present a relational perspective that investigates the socioenvironmental interactions of populations migrating due to forced displacement, applying social network analysis (SNA) to understand the socioenvironmental shaping of flood risk in precarious settlements using the example of a case in Colombia. We meet networks of migrants who create precarious settlements in environmentally protected areas with a precarious perception of risk - when desperate and faced with a lack of opportunities or access to land for construction purposes- who create informal institutional links that sustain the development of the settlement with political favours. Recognising the formation of these networks provides an opportunity to reflect on their ways of thinking and acting. Moreover, they represent an important source of information to propose dialogue scenarios involving the relevant actors, which may in turn facilitate the definition of collective agreements to construct safer and more sustainable environments.
\end{abstract}

Key words: Migration; social and environmental networks of the place; analysis; socioenvironmental shaping of flood risk; precarious settlements.

\footnotetext{
Universidad Industrial de Santander. Colombia amorocho@uis.edu.co

2 Universidad Externado de Colombia. Colombia

dolly.palacio@uexternado.edu.co
} 
Sumario: Introducción. 1. Antecedentes teóricos del Trabajo Social en la perspectiva ambiental. 2. Las redes socioambientales del lugar: perspectiva teórica y metodológica. 3. Un estudio de caso en Colombia: La configuración social del riesgo ambiental en el Río de Oro - Girón. 3.1. La red de inmigrantes pioneros que fundaron el asentamiento precario Las Marías. 3.2 Las redes socioambientales e institucionales que favorecieron la consolidación del asentamiento precario Las Marías. 4. Conclusiones. 5. Referencias bibliográficas.

Cómo citar: Amorocho Pérez, A. M.; Palacio Tamayo, D. C. (2018) El Trabajo Social relacional: una perspectiva para rastrear la configuración socioambiental del riesgo de inundación en los asentamientos precarios, en Cuad. trab. soc. 31(2), 467-478.

\section{Introducción}

La construcción social del riesgo se ha estudiado con alguna profundidad y con un énfasis en las disciplinas de geografía, sociología y urbanismo desde los años noventa (Pliego, 1994; Lavell y Franco, 1996; Beck, 1998; Ríos, 2005) y más recientemente desde una perspectiva histórica (Serna, 2010). También se ha demostrado la relación entre pobreza y desastres (Fothergill y Peek, 2004). El Trabajo Social, por su parte, ha participado con su hacer en la atención humanitaria de estos eventos, proponiendo acciones de atención psicosocial y de redes de apoyo (Dominelli, 2014; Pyles, 2015; Pyles, 2017).

En este artículo evidenciamos que más allá de los factores estructurales generales, los desastres por inundación en asentamientos urbanos en los contextos informales son resultado de una configuración socioambiental del riesgo que articula una compleja red de interacciones entre personas que migran en cadena, con lazos de parentesco o amistad, hacia un territorio que ofrece elementos ecológicos para la autoconstrucción de viviendas precarias. Este fenómeno en Colombia obedece principalmente a dos factores relacionados. El primero es la configuración de un comportamiento colectivo que sufre de una desconexión perceptual con el entorno ecológico que resta importancia al potencial destructivo del río, en caso de presentarse un evento meteorológico extremo, como las lluvias torrenciales. El segundo se refiere a la configuración de vínculos institucionales por parte de los fundadores que se tejen de manera informal y, basados en los favores políticos, contribuyen a la consolidación del asentamiento en oposición a las normas urbanísticas y ambientales.
Con el objetivo de aportar, desde la perspectiva del Trabajo Social, a la identificación de factores que pueden hacer una detección temprana y prevenir el riesgo de desastres relacionados con eventos naturales, en este artículo proponemos recuperar las bases de su teoría social sobre la relación entre "la persona y su entorno" que está en los fundamentos teóricos del Trabajo Social, desde Octavia Hill, Jane Addams y Mary Richmond, para articular estas nociones a las perspectivas relacionales contemporáneas como el análisis de redes sociales, con el fin de aportar a la comprensión de la estructuración de los vínculos que construyen socialmente el riesgo.

Para ello, dividimos este artículo en cuatro partes. La primera presenta los antecedentes del Trabajo Social en la perspectiva ambiental y sus aportares para entender y atender los problemas del riesgo de inundaciones. En la segunda, presentamos los aspectos teóricos y metodológicos de las redes socioambientales del lugar y su contribución al Trabajo Social ambiental, dialógico y situado. La tercera ilustra un estudio de caso en Colombia, la aplicación del análisis de redes sociales (ARS) para visualizar la redes de inmigración, las interacciones socioambientales del lugar y socio-institucionales, su potencia para generar investigaciones diagnósticas que permitan identificar la configuración socioambiental del riesgo. La última parte, a manera de conclusión, reflexiona sobre la importancia de analizar las propiedades de las redes como la adyacencia y la centralidad, como componentes relacionales que configuran la vulnerabilidad relacional, aspecto central para enfrentar los desafíos de la configuración socioambiental del riesgo de inundaciones y el desarrollo de propuestas de manejo sostenible del lugar. 


\section{Antecedentes teóricos del Trabajo Social en la perspectiva ambiental}

El Trabajo Social desde sus inicios señala la importancia del entorno (natural y construido) en el bienestar humano, abordando de manera sistemática la pregunta sobre la relación entre "la persona y su entorno" (Hill, 1877; Addams, 2013 [1892]; Richmond, 2005 [1917]). En sus orígenes, el debate en Trabajo Social sobre esta relación planteó tres posturas centrales. Una a mediados del siglo XIX: Octavia Hill creó un sistema que consistió en la mejora de las condiciones de vida de las casas de renta habitadas por las familias obreras en Londres (Muxí, 2015). Además, propuso crear lugares verdes abiertos para el disfrute de los habitantes de áreas marginales de esta ciudad, con el fin de que tuvieran acceso al aire libre, el esparcimiento y disfrute de la naturaleza. Su propuesta propició la fundación The National Trust3 y la Sociedad de Espacios Abiertos en Inglaterra (Hill, 1877). Dos, la propuesta de Jane Addams en 1892, promotora del Settlement House Movement, dio importancia al entorno social para alcanzar el bienestar humano, entendido el ambiente en un sentido más amplio, como el "entorno urbano". Además del entorno social, el entorno de vida también contiene el entorno físico y construido (condiciones de salubridad de la vivienda, contaminación, etc.), y servicios locales (Addams, 2013). Tres, hacia 1917 Mary Richmond se propuso establecer el diagnóstico social desde la perspectiva de las relaciones de la persona con su entorno (social y espacial) para definir su situación (Richmond, 2005) y buscar caminos de intervención para lograr el bienestar de la persona. Esta pionera del Trabajo Social propuso el concepto de "humano-en-medio ambiente" que contemplaba principalmente los elementos sociales, más no los naturales.

En años posteriores, durante la mayor parte del siglo XX, esta preocupación por la relación entre la persona y su entorno se enfocó en la persona y su medio ambiente social (McKinnon, 2008), dejando sin desarrollar la relación con los ambientes naturales que planteó Octavia Hill. Según McKinnon (2008), durante muchos años los trabajadores sociales se han comprometido con el concepto de "medio ambiente", pero entendiéndolo casi exclusivamente como entorno sociocultural o psicoso- cial. Dejando casi inexplorado el ambiente entendido como sistema ecológico, descuidando en la teoría y la práctica del Trabajo Social la interacción entre los seres humanos y el resto del mundo natural.

A partir de la década de los años noventa del siglo pasado, se retoman los planteamientos de esta relación, incorporando los procesos naturales en el desarrollo humano, y construyendo una perspectiva que relaciona el bienestar humano y el bienestar de la tierra (Hoff y McNutt, 1994). Las propuestas que en adelante se desarrollan retan al Trabajo Social a participar en el debate público sobre el fenómeno ambiental en las sociedades modernas (Närhi, 2004).

Por su parte, Germain y Gitterman entendieron que los seres humanos actúan dentro del medio ambiente físico, la sociedad y la cultura. El entorno físico incluye el mundo natural, las estructuras construidas por la gente, el espacio que apoya, contiene, o arregla esas estructuras, y los ritmos de la biología humana y ambiental (1995).

Asimismo, Gray y Coates (2012) se cuestionan sobre en qué medida, cómo y por qué los trabajadores sociales deberían involucrarse en cuestiones geopolíticas fundamentales como: la preocupación por el cambio climático, el calentamiento global, la degradación del medio ambiente, la contaminación, la contaminación química, la agricultura sostenible, la gestión de desastres, entre otras. Además, se preguntan sobre el significado de lo "no-humano" para el Trabajo Social.

Los diversos problemas ambientales, como el cambio climático y los desastres naturales, han demandado la intervención del Trabajo Social. Esto se evidencia en la atención humanitaria durante y después de los desastres realizada por trabajadores sociales; por ejemplo, el trabajo realizado con organizaciones rurales campesinas en Haití para desarrollar capacidades en la investigación acción participativa y la recuperación y desarrollo sostenible de la comunidad después del terremoto de 2010 (Pyles, 2015).

Además, los trabajadores sociales se han interesado por examinar las perspectivas $\mathrm{y}$ actitudes de las comunidades afectadas por el cambio climático en Canadá, y el papel que juega el desarrollo social en relación a la adaptación y respuesta frente a este fenómeno 
(Drolet y Sampson, 2017) y han considerado el género como un factor clave en la vulnerabilidad a los desastres (Alston, 2013).

También han adelantado algunas investigaciones, como la realizada por Dominelli entre 2009 y 2012 sobre el tsunami del Océano Índico de 2004 en el suroeste de Sri Lanka, que examinó las experiencias de los sobrevivientes de desastres en materia de ayuda humanitaria y los procesos de reconstrucción (2014).

Actualmente se reconoce la acción de los trabajadores sociales en entornos de desastre en todo el mundo y colaboran con diversos actores, como las organizaciones humanitarias; sin embargo, se hace una crítica a que muchas de las intervenciones de desastres pueden contribuir a perpetuar unas prácticas colonialistas, neoliberales y de opresión, por lo que se recomienda descolonizar la práctica del Trabajo Social en casos de desastre (Pyles, 2017).

El presente artículo busca aportar al desarrollo del Trabajo Social ambiental, planteándose un enfoque para develar las interacciones entre las personas y su ambiente, proponiendo Las redes socioambientales del lugar como una perspectiva relacional, dialógica y situada, retomando los desarrollos de Palacio (2001, 2002, 2015 y 2017), para la investigación diagnóstica en Trabajo Social referida a la configuración socioambiental y aplicarla a la configuración del riesgo de desastres por inundación.

En el trabajo de Palacio (2017) se hace hincapié en tres ejes centrales:

1. La relación de las personas en su entorno se amplía más allá de sus relaciones sociales, hasta reconocerlas como parte de los entornos naturales, donde los seres vivos no humanos y los elementos naturales que forman parte del entorno son fundamentales para el desarrollo de la persona. Por ejemplo: el agua, la tierra, los animales y plantas que hacen posible que la persona mantenga su vida directa e indirectamente;

2. Los elementos de la relación entre persona-entorno ambiental se contemplan desde una perspectiva relacional; es decir, se entiende que las personas usan, se apropian de, valoran y organizan ese entorno de tal manera que la composición, la estructura y la dinámica ecológica del lugar tiene efectos sobre el bienestar o malestar de las personas y sus entornos y viceversa;
3. Reconocer estas relaciones socioambientales por parte de las comunidades y de los trabajadores sociales es importante para crear diálogos situados que favorezcan el despertar de una consciencia y una práctica ambiental que reduzca los niveles de desconexión perceptual de las comunidades frente a su entorno socioambiental, aportando a la gestión de riesgos ambientales y al bienestar.

\section{Las redes socioambientales del lugar: perspectiva teórica y metodológica}

Esta perspectiva se ha abordado en el marco de la gestión participativa de las áreas protegidas (Palacio, 2002, 2003), el estudio de la construcción social del riesgo (Amorocho, 2008, 2010, 2012), y la gobernanza de los ecosistemas estratégicos (Palacio, 2015). En este trabajo se presenta esta perspectiva como parte de un enfoque teórico-práctico para el Trabajo Social en lo referido a lo que hemos denominado: configuración socioambiental del riesgo. Se reconoce que la investigación diagnóstica para el Trabajo Social permite, además del encuadre de situaciones problema, la construcción de procesos reflexivos colectivos sobre los cuales conocer los modos de actuar, de percibir e interpretar las prácticas sociales en entornos particulares, para identificar y proponer nuevos formas de acción e interacción socioambiental (Palacio, 2002).

"El lugar" y "la red" no son términos fortuitos. Primero de todo, la decisión de ubicar el Trabajo Social en el lugar no es una decisión localista. Por el contrario, comprender y crear reflexión sobre la configuración del "lugar" implica reconocer tanto los entramados locales y sus dislocaciones, como los globales localizados en el lugar (Latour, 2008). Adicionalmente, el trabajador social en esta perspectiva está involucra en las relaciones del lugar, debido a que como investigador se implica (Villasante y Gutiérrez, 2006) con los sujetos de la intervención, y todos se definen como actores que intentan influir en el ensamblaje del mismo, según Latour. El lugar, a su vez es producto de las prácticas de todos los actores humanos y no humanos que actúan e interactúan directa e indirectamente en él (Massey, 1997; Thrift, 1997). Dicha aproximación al lugar y sus redes socioambientales tiene el objetivo de 
constituirse en un punto de partida para el conocimiento de los procesos de ensamblaje del lugar, para generar procesos reflexivos entre todos los implicados, a partir de sus relaciones con el lugar, con el fin de generar transformaciones orientadas hacia la sostenibilidad de la vida (Palacio, 2017).

En síntesis, las relaciones sociales no son relaciones entre seres humanos solamente; estas comprometen de manera mucho más directa y menos consciente, "el entorno o ambiente". El entorno, por su parte es un ensamblaje o red socioambiental. Es decir, el entorno es el resultado de patrones de asociación entre humanos y otras entidades (naturales y culturales) que se forman a partir de las interdependencias entre conjuntos de seres (especies y sus poblaciones incluyendo al ser humano), elementos naturales (aire, agua, tierra, fuego, etc.) y objetos culturales (artefactos elaborados por los humanos) en segmentos de tiempo y espacio. Estos vínculos pueden ser más o menos permanentes, y son los que dan origen a la organización de las dinámicas socioambientales en La Tierra.

En este sentido, atendiendo a la teoría de las redes sociales, sobre la difusión y la influencia (Chistakis y Fowler, 2009), los significados que se van formando en las personas y el entorno depende de los vínculos que las configuran, lo que influye tanto en la formación de una conciencia ecológica como de una cierta desconexión perceptual del entorno según las adyacencias que se configuran en la red. Es decir, el grado de cercanía entre actores y sus formas de pensar y de hacer, puede influenciar las maneras de pensar y hacer que se generalizan en la red socioambiental del lugar, construyendo un patrón de orden o caos en el sistema $\mathrm{y}$, por lo tanto, empoderamiento o riesgo que sostienen o deteriora el entorno socionatural.

Esta perspectiva relacional puede hacerse operativa para reconocer los patrones de relación entre conjuntos determinados de actores y sus entornos mediante la aplicación de métodos provenientes del análisis de redes sociales (Wasserman y Faust, 1994). El análisis de redes sociales como paradigma epistemológico nace en los años cuarenta del siglo XX, pero sus mayores avances se han hecho en los últimos veinte años con el desarrollo de la informática (Freeman, 2004, p. 205). En el caso que se presenta, se exponen las principales herramientas de análisis (Wasserman y Faust, 1994), entre ellas, las sociomatrices, construidas en matrices de excel y graficadas usando UCINET VI (Borgatti, Everett y Freeman, 2002).

Para captar las relaciones que guardan la migración con la conformación de los asentamientos precarios y a la vez reconocerlos como lugares-red, configurados a partir de la interacción de la red de migratoria y los vínculos socioambientales e institucionales, se requirió una propuesta metodológica que inicialmente permitiera descubrir el establecimiento y funcionamiento de la red de migración. Para explicar la red de inmigración se retomaron los planteamientos de la teoría de redes migratorias (Massey et al.,1998 citado en García, 2001). Esto se logró entendiendo que el asentamiento precario ubicado en el cauce del río fue fundado por personas que migraron a ese territorio con el fin de acceder al suelo y otros elementos ecológicos, los que usaron y apropiaron para construir una vivienda.

La estrategia metodológica incluyó definir el límite espacial y temporal en el que se han configurado los asentamientos en el cauce del río de $\mathrm{Oro}^{4}$, siendo necesario la ubicación cartográfica, de fotografía satelital y las visitas a los asentamientos. Se seleccionó a Las Marías teniendo en cuenta dos criterios: localización del asentamiento en el cauce del río de Oro y el tiempo de conformación.

Una vez elegido el asentamiento a estudiar y conociendo su crecimiento poblacional, se decidió realizar el trabajo de campo, bajo la perspectiva de buscar hombres y mujeres (inmigrantes pioneros sin contacto previo o con él, y/o nativos) que cumplieran con la característica de ser fundadores/as de los asentamientos seleccionados, lo que implicó que hubiese llegado durante los dos primeros años de haberse constituido.

Los sujetos inmigrantes fundadores del asentamiento estudiado se identificaron siguiendo el método bola de nieve: 21 personas (17 mujeres y 4 hombres) inmigrantes pioneros, a quienes se realizó una entrevista semiestructurada. A partir de las entrevistas realizadas, se conocieron las características sociodemográficas de la población. También se tomó información respecto a la vivienda antes y después de llegar al asentamiento, así como información sobre el entorno ecológico y los efectos de la inundación ocurrida en 2005.

\footnotetext{
Se identificaron ocho.
} 
Para reconstruir las red de inmigración, así como las interacciones de los inmigrantes con los elementos ecológicos del entorno y con los actores institucionales, se hizo uso de las "matrices de afiliación" o "matrices de dos modos", que se caracterizan por representar a los actores y su participación en determinados eventos, generalmente membresías o interacciones con entidades sociales o no humanas, o con lugares geográficos, etc. Algunas de las preguntas que ayudaron a construir estas matrices de afiliación fueron, para el caso de la red de inmigración: la relacionada con pedirle al entrevistado que mencionara las personas que conocía que vivían en el asentamiento al momento de su llegada $\mathrm{y}$, además, se le preguntó si invitó a algún pariente, amigo o conocido a vivir en Las Marías. Para el caso de las interacciones socioambientales: las relacionadas con pedirle al entrevistado que enumerara los elementos de la naturaleza que recordaban estaban en el territorio cuando llegó allí y el uso que les daba. Para reconstruir los vínculos socio-institucionales se plantearon las siguientes preguntas: ¿cómo se fue accediendo a los servicios públicos?, ¿cómo fue el proceso de construcción de carreteras, Iglesia, salón comunal, $u$ otro tipo de infraestructura comunitaria?, ¿qué personas/entidades ayudaron a construir esa infraestructura? y ¿en qué año fue eso?

A continuación se presenta un estudio de caso que evidencia cómo se da la configuración socioambiental del riesgo cuando se construyen viviendas precarias en el cauce de un río en el sector urbano de un municipio. Esto amerita contar con una perspectiva relacional que devele el proceso de inmigración y asentamiento sobre el territorio, así como los vínculos socioambientales e institucionales que favorecieron la consolidación de un asentamiento precario y sus respectivas redes de lugar.

\section{Un estudio de caso en Colombia: La configuración social del riesgo ambiental en el Río de Oro - Girón}

El caso que se presenta en este apartado corresponde a una investigación -realizada entre 2007 y 2008 - que devela las redes de lugar que favorecieron la configuración socioambiental del riesgo de inundación en el cauce del Río de Oro que llevó a desencadenar un desastre en febrero de 2005 en el municipio de Girón ${ }^{5}$, época en la que hubo un período de fuertes lluvias que produjeron una inundación de gran magnitud que dejó 10 víctimas fatales, 3.280 familias damnificadas, 2.700 viviendas destruidas y 580 averiadas (Observatorio de Salud Pública de Santander, 2005). Esta inundación fue altamente destructiva en el cauce del Río de Oro y afectó principalmente a los asentamientos precarios que se ubicaban allí.

La identificación de la redes migratorias, socioambientales y socio-institucionales que configuraron el asentamiento informal estudiado se hizo mediante el análisis de redes sociales, que permitió ver el enlace entre los actores inmigrantes fundadores de Las Marías y entre estos y su entorno institucional y socioambiental, para el acceso al suelo, al agua, la infraestructura urbana y los servicios públicos. El trabajo permitió, a partir del uso de gráficos y matrices (Wasserman y Faust, 1994), representar lo que se denominó red de inmigración de los fundadores del asentamiento, las interacciones socio-ambientales para el acceso a elementos ecológicos como el agua y el suelo y los vínculos socio-institucionales que hicieron posible su consolidación. Estas interacciones construyeron un asentamiento informal y configuraron un escenario de riesgo por inundación.

\subsection{La red de inmigrantes pioneros que fun- daron el asentamiento precario Las Marías}

En Colombia la debilidad de la política pública de vivienda no logra garantizar viviendas sociales a la población de bajos ingresos migrante campesina ni a la desplazada por la violencia política, que llega a la ciudad y que no cuenta con ingresos suficientes y estables para adquirir o alquilar una vivienda vía mercado inmobiliario.

Es por eso por lo que se fundan asentamientos en zonas de fragilidad ambiental como las rondas de los ríos en los sectores urbanos de las ciudades colombianas, como se evidenció en el asentamiento Las Marías, dado que de los 21 entrevistados, el 76 por ciento mencionaron que no contaban con vivienda propia antes de llegar al lugar. Los primeros habitantes que se asentaron en ese territorio lo hicieron porque

Municipio perteneciente al departamento de Santander, ubicado al noreste de Colombia. Tiene una superficie de $475.14 \mathrm{~km}^{2} \mathrm{y}$ en el Censo de 2005, se reportó una población total de 135.531 habitantes. 
consideraron que requería acceder al suelo para autoconstruir una vivienda, así fuera precaria, y eligieron el terreno que corresponde al cauce del Río de Oro que atraviesa el casco urbano del municipio de Girón. Este suelo está clasificado en la categoría de protección forestal y de amenaza de inundación, según la legislación ambienta $1^{6} \mathrm{y}$ urbanística colombiana ${ }^{7}, \mathrm{y}$ no es apto para la construcción de viviendas ni otro tipo de edificación ${ }^{8}$.

La configuración de este asentamiento informal fue propiciada por la migración de personas hacia ese territorio constituyendo una red de inmigración que muestra un patrón de llegada y permanencia en el lugar, a partir de unos actores-inmigrantes pioneros que construyeron las primeras viviendas precarias y luego lograron atraer a otros familiares y amigos.

El primer momento de conformación del asentamiento Las Marías se da cuando en 1988 llegó una mujer desplazada por la violencia del sector rural del municipio de Sabana de Torres ${ }^{9}$ junto a su familia. Esta mujer invitó a un paisano a asentarse en el lugar, quien también había vivido el desplazamiento forzado y este invitó a algunos parientes más. Durante esa época, este sector era suburbano.

El segundo momento inicia con la identificación de la llegada de otra mujer al lugar en 1996 y junto con ella llegan 15 familias $^{10}$ más que se consideraron a sí mismas como fundadoras del asentamiento. La mayoría de estas familias vivían en alquiler en un barrio precario ubicado cerca al cauce del río, aunque previamente la mayoría de ellas habían migrado desde el sector rural. Específicamente se encontró que de los 21 entrevistados el 43 por ciento vivía en pieza en alquiler y 29 por ciento en casa en arriendo, en el domicilio anterior. Esto pobladores fueron los que posteriormente fueron afectados por la inundación que aconteció en el 2005. En el Gráfico 1 se muestra cómo se fue tejiendo esta red de inmigración a partir de vínculos familiares y sociales.

Gráfico 1. Red de Inmigración que configuró el asentamiento Las Marías.

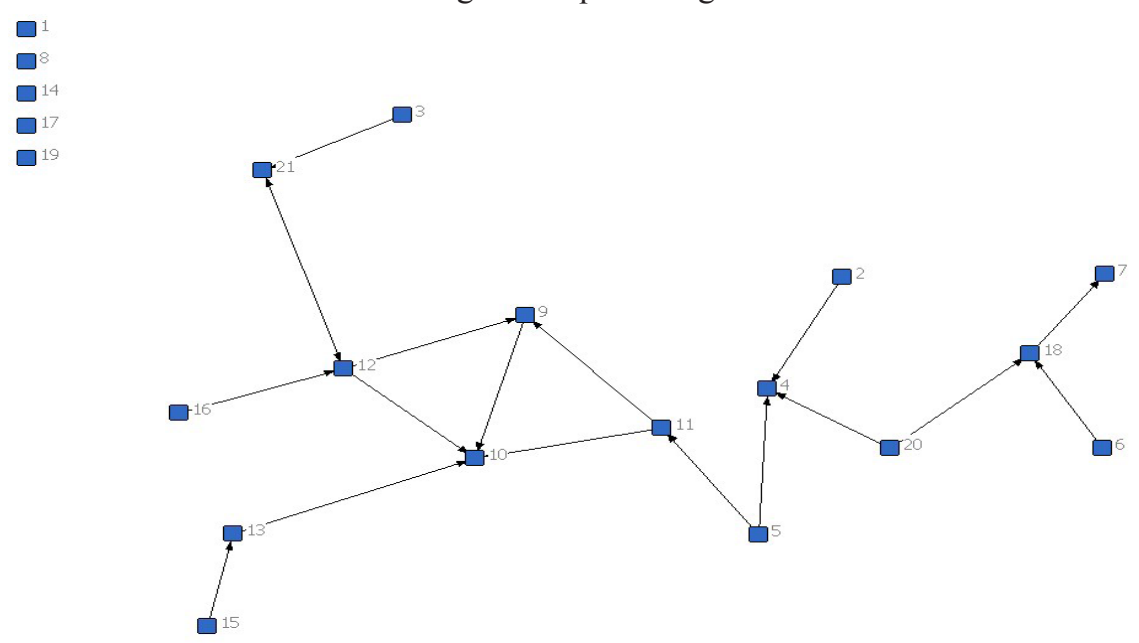

Fuente: Amorocho, 2008, 2012.

Nota: Este gráfico se construyó con base en una matriz de modo simétrico para observar la propiedad "conocer a" antes de llegar al asentamiento estudiado. Se mide la ausencia o presencia de la relación; en el caso en estudio si la persona A afirma conocer a B se le da el valor de 1 , y si no, 0.

$6 \quad$ Ley 2811 de 1974 (Código Nacional de Recursos Naturales Renovables y de Protección al Medio Ambiente) y Ley 388 de 1997 (Ley de Ordenamiento Territorial).

$7 \quad$ Ley 9 de 1989 (Ley de Reforma Urbana) y el Decreto 564 de 2006 (Reglamenta las disposiciones relativas a las licencias urbanísticas, al reconocimiento de edificaciones, a la función pública que desempeñan los curadores urbanos y a la legalización de asentamientos humanos constituidos por viviendas de interés social).

8 En Colombia las viviendas construidas en los cauces de río en la ciudad no pueden ser legalizadas porque están en zonas de amenaza y riesgo no mitigable y en suelos de protección, según lo estipula la Ley 388 de 1997 y el Decreto 564 de 2006.

$9 \quad$ Municipio colombiano perteneciente al departamento de Santander.

10 Entrevista líder comunitario Asentamiento Las Marías, 2007 
El asentamiento Las Marías se fundó a partir de vínculos familiares (40 por ciento) y de amistad (30 por ciento). En la red de inmigración del asentamiento Las Marías (Gráfico 1) el actor central es la mujer identificada con el número 10, que migró del sector rural del municipio de Sabana de Torres, como se mencionó anteriormente fue desplazada por la violencia política, y se asentó en ese territorio en el año de 1988. Cuatro de los 21 entrevistados la reconocen como fundadora, amiga o pariente, siendo la que construyó la primera vivienda precaria en una parcela de vocación agrícola que compró a un tercero. (Amorocho 2008, 2012)

La mujer que se identifica en la red de inmigración con el número 4 es el otro actor central de la red. Nació en el sector rural de Girón y pertenece a una de las 15 familias que en 1996 se asentaron en el lugar. El actor 9 es un hombre que migró desde el sector rural de Sabana de Torres y era conocido del actor 10. Los actores 12 y 21 corresponden a dos mujeres que vivieron en otro asentamiento precario en el mismo municipio justo antes de invadir el cauce del Río de Oro en que se fundó las Marías. El actor 18 es una mujer procedente de un barrio formal ubicado muy cerca de Las Marías. En Las Marías un buen número de personas son familiares de los actores 9 y 21 . Los sujetos mencionados son los actores prominentes de la red de inmigración que corresponde con los fundadores de este asentamiento (Amorocho 2008, 2012).

\subsection{Las redes socioambientales e institucio- nales que favorecieron la consolidación del asentamiento precario Las Marías}

Entre los hallazgos de la investigación se observó que el significado ambiental del Río de Oro no es reconocido por la mayoría de los fundadores de Las Marías, prácticamente no se nombra en las entrevistas, cuando se pregunta por los elementos que recuerdan cuando llegaron al lugar; por lo tanto, la existencia del río está invisibilizada. La fundadora que llegó al lugar en 1988 sí recuerda haber bebido agua del río, bañarse en él y haber lavado ropa en sus aguas. Ella, y su familia, recuerdan el lugar como un sitio agradable, donde el agua del río era limpia se podía pescar y era lugar de recreación para los habitantes del municipio de Girón. Sin embargo, esto no lo reconocen algunas de las mujeres pertenecientes a las 15 familias fundadoras del asentamiento que llegaron en 1996. Los fundadores posteriores sí lo reconocen cuando se les pregunta explícitamente sobre la evacuación de aguas servidas de origen doméstico, lo que muestra una visión de río como recurso.

De igual modo se identificó que la mayor contaminación del río inicia hacia la década de los noventa, por eso los inmigrantes pioneros del asentamiento Las Marías, mencionan que hacia 1988 el río aún estaba limpio. Posteriormente, a partir de 1991, ellos recuerdan que se empiezan a reportar malos olores emanados del río, situación relacionada con la creación de la zona industrial de Chimitá durante la década de los años noventa.

En la población estudiada, se evidencia una baja consciencia ambiental. La carencia de infraestructura de servicios públicos y de una red de alcantarillado, asume al río como el canal de evacuación de las aguas servidas. Entre los 21 entrevistados se encontró solo una persona que usó un sistema de letrina para evacuar las aguas servidas de origen doméstico. Esta persona es la mujer fundadora del asentamiento Las Marías, quien afirmó usar esta tecnología a finales de los años ochenta para no contaminar las aguas del río; no obstante, tiempo después, ella dejó de usar este sistema para, al igual que sus vecinos, evacuar las aguas servidas directamente en él.

Respecto a los otros elementos ecológicos, estos sí eran recordados por la mayoría de los fundadores. Particularmente recuerdan existían en el lugar árboles de mango, mamón, limones, naranja, entre otros. También se sabe que los primeros pobladores criaban cerdos, práctica rural que subsistió durante largo tiempo. Es necesario anotar que el terreno donde se fundó el asentamiento Las Marías, correspondía a suelo rural hasta la llegada de los inmigrantes fundadores que, junto a otros actores, lo urbanizaron informalmente (Amorocho, 2008).

Por otra parte, las interacciones socio-institucionales que aportaron a la consolidación del asentamiento. Se encontró que los inmigrantes buscaron el apoyo de los políticos de turno, iglesias, las organizaciones sociales y empresas públicas para acceder a materiales de construcción, servicios públicos e infraestructura comunitaria consolidando con su ayuda el asentamiento. Estos vínculos les facilitaron el acceso informal al agua potable (inicialmente pila pública) y posteriormente el acceso a ser- 
vicios públicos (energía eléctrica, gas, recolección de basuras), construcción de infraestructura urbana (vías e instalación de desagües de aguas servidas con descarga al río).

Para las personas que llegaron posteriormente al asentamiento Las Marías, el acceso al territorio se da por compra o alquiler de la vivienda, las cuales resultan atractivas al tener las conexiones a servicios públicos, especialmente agua potable, garantizando su disponibilidad por las conexiones informales a la red de acueducto, realizadas por los primeros pobladores.

Las alianzas que han mantenido los habitantes de Las Marías con los actores institucionales, sociales y estatales, son inestables y empiezan a resquebrajarse a partir de 1997, cuando se establece la Ley 388 que obliga a los municipios a elaborar el Plan de Ordenamiento Territorial ${ }^{11}$; en el cual se definen las rondas de río como suelos de protección al mismo tiempo que se declaran como zona de amenaza por inundación y, por tanto, zonas en alto riesgo de desastre. Dada la ubicación de estos asentamientos precarios, previa la sanción de esta Ley, el proceso de consolidación de estos asentamientos se empezó a desestimular, especialmente a partir de la inundación de 2005, configurándose los conflictos entre los habitantes de esos lugares y el gobierno local, con algunos intentos de desalojo a partir del uso de la fuerza pública.

\section{Conclusiones}

Este trabajo muestra que los desastres por inundación en asentamientos precarios son resultado de la configuración socioambiental del riesgo que articula la migración de personas, con lazos de parentesco o amistad, con un territorio que ofrece elementos ecológicos para la autoconstrucción de viviendas. En esta configuración de vecindario socioambiental se generaliza una desconexión perceptual de los migrantes con el entorno ecológico que desestima la fuerza hidráulica del río en caso de presentarse una lluvia torrencial. De igual manera se muestra claramente cómo se establecen los vínculos socio-institucionales que coadyuvaron a la consolidación del asentamiento, a partir de facilitar las conexiones informales de servicios públicos y el mejoramiento de infraestructura física. Todo ello en oposición a la normativa ambiental y urbanística en Colombia.

Tomar en consideración la perspectiva relacional y situada para realizar las investigaciones diagnósticas en Trabajo Social permitió comprender mejor fenómenos complejos como los desastres por inundación en el cauce de un río de un municipio intermedio. En el caso presentado se puede observar que, cuando existe un inadecuado acoplamiento entre las personas, el ambiente natural y las instituciones, se puede alterar su bienestar, el de su familia, comunidad y el del entorno natural. Esto evidencia la debilidad de la política de vivienda social y la baja gobernanza sobre el territorio..

Este estudio aporta evidencias para responder a la pregunta planteada por Gray y Coates sobre ¿cuál es el significado de lo "no-humano' para el trabajo social? (2012), pues al partir de la relación entre la "persona y su entorno", yendo más allá de los aspectos sociales e incluyendo los elementos naturales, fue posible ver la interrelación entre humanos y no humanos que lograron un acoplamiento inadecuado, cuando a partir de esas relaciones socioambientales y los vínculos institucionales se generaron un escenario de riesgo que terminó en un desastre por inundación.

En este trabajo se evidenció que el entendimiento del riesgo de desastres requiere asumir las interacciones entre humanos y no humanos, lo que podría aportar a una mejor comprensión del riesgo de desastres planteada en la Prioridad 1 del Plan de Acción del Marco de Sendai. En ese Plan de Acción se plantea que la gestión del riesgo de desastres debe basarse en una comprensión del riesgo de desastre en todas sus dimensiones de vulnerabilidad, capacidad, exposición de personas y bienes, características de peligro y el medio ambiente. Estos conocimientos pueden utilizarse para la evaluación de riesgos, prevención, mitigación, preparación y respuesta (Wahlstrom, 2017).

Metodológicamente este estudio realiza un aporte al Trabajo Social ambiental, al asumir los asentamientos informales precarios desde la noción teórica del lugar-red y al hacer uso del análisis de redes sociales para visualizar la red de inmigración y la teoría del actor-red

11 El cauce del Río de Oro del sector urbano de Girón fue declarado como zona de alta amenaza de inundación en el Plan de Ordenamiento Territorial (POT) de este municipio en el 2000. 
(Latour, 2008, p. 390.) para evidenciar las conexiones socio-institucionales que ayudaron a consolidar el asentamiento. Esta herramienta de investigación diagnóstica que incluye elementos demográficos como la migración, unido a los vínculos socioambientales y socio-institucionales que articulados configuraron un escenario de desastre socionatural, resultan interesantes a la hora de establecer planes de prevención y atención del riesgo.

Esta mirada es un ensamblaje que visualiza las redes de inmigración, socioambientales y socio-institucionales, las que evidencian las formas de ejercicio del poder de actores sobre los elementos ecológicos contenidos en el territorio en un tiempo específico, es decir la construcción de un "lugar-red" (Palacio, 2001).

En síntesis, la composición y la estructura de las redes y la información sobre los procesos de su estructuración son una fuente de información importante para construir procesos de diálogo y participación en los procesos de planeación y ordenamiento territorial de los lugares mismos donde ocurren dichas interacciones. Estas evidencias pueden servir de base a la reflexión entre los actores mismos sobre sus vínculos para profundizar en las posibilidades de armonización de estas relaciones, para una mayor sostenibilidad de la vida en el territorio.

\section{Referencias Bibliográficas}

Addams, J. (2013[1892]). El valor objetivo de un centro social. En: J. Addams, A. Lima y C. Verde, Hull House. El valor de un centro social (pp. 61-74). Madrid: Paraninfo y Consejo General de Trabajo Social.

Alston, M. (2013). Environmental Social Work: Accounting for Gender in Climate Disasters. Australian Social Work, 66(2), 218-233. Doi:10.1080/0312407X.2012.738366

Amorocho, A. (2008). Interacciones entre la inmigración, la construcción de territorios y los desastres naturales: El caso de la inundación del río de Oro en Girón, Santander. (Tesis de Maestría, Bogotá: Universidad Externado de Colombia).

Amorocho, A. (2010). La construcción de asentamientos humanos precarios en zonas de inundación y la conservación de cauces de río. Prospectiva, 15, 295-328. Disponible en: http://revistaprospectiva.univalle.edu.co/index.php/prospectiva/article/view/1113/1232

Amorocho, A. (2012). Redes de inmigración, asentamientos informales y zonas de riesgo de inundación: el caso del Río de Oro en Santander (Colombia). Territorios 26, 13-33. Disponible en: http://revistas. urosario.edu.co/index.php/territorios/article/view/2116

Beck, U. (1998). Políticas ecológicas en la edad del riesgo. Antídotos. La irresponsabilidad organizada. Barcelona: El Roure.

Borgatti, S., Everett, M. y Freeman, L. (2002). Ucinet for Windows: Software for Social Network Analysis. Harvard Analytic Technologies.

Chistakis, N. y Fowler, J.H. (2009). Connected. The Surprising Power of Our Social Networks and How They Shape Our Lives. Nueva York: Little Browon and Company.

Dominelli, L. (2014). Promoting environmental justice through green social work practice: A key challenge for practitioners and educators. International Social Work, 57(4), 338-345. Doi: $10.1177 / 0020872814524968$

Drolet, J., \& Sampson, T. (2017). Addressing climate change from a social development approach: Small cities and rural communities' adaptation and response to climate change in British Columbia, Canada. International Social Work, 6 (1), 61-73. Doi: 10.1177/0020872814539984

Fothergill, A. y Peek, L. (2004). Poverty and disasters in the United States: A review of recent sociological findings. Natural Hazards, 32(1), 89-110. Doi: 10.1023/B:NHAZ.0000026792.76181.d9

Freeman, L. (2004). The Development of Social Network Analysis. A Study of Sociology of Science. Vancouver: Empirical Press.

García, R. (2001). El papel de las redes migratorias en las migraciones a corta y media distancia. Scripta Nova. Revista Electrónica de Geografía y Ciencias Sociales (Número extraordinario). Disponible en: http://www.ub.edu/geocrit/sn-94-11.htm

Germain, C. y Gitterman, A. (1995). The Life Model of Social Work Practice. Advance in Theory \& Practice. Second Edition. Nueva York: Columbia University Press. 
Gray, M. y Coates, J. (2012). Environmental ethics for social work: Social work's responsibility to the non-human world. International Journal of Social Welfare, 21(3), 239-247. Doi: 10.1111/j.14682397.2011.00852.x

Hill, O. (1877). Our common land and other short essays. Londres: MacMillan and Co.

Hoff, M. y McNutt, J. (1994). The global environmental crisis: implications for social welfare and social work. Aldershot: Avebury.

Latour, B. (2008). Reensamblar lo social. Una Introducción a la Teoría del Actor-Red. . Buenos Aires: Ediciones Manantial.

Lavell, A. y Franco, E. (1996). Estado, Sociedad y Gestión de los Desastres en América Latina: En Busca del Paradigma Perdido. Lima: La Red-FLACSO. Recuperado de: http://www.desenredando.org/public/ libros/1996/esyg/esyg_Intro_dic-18-2002.pdf

Massey, D. (1997). A Global Sense of Place. En: T. Barnes y D. Gregory, Reading Human Geography. The poetics and politics of Inquiry (pp. 315-323). Londres: Arnold.

McKinnon, J. (2008). Exploring the Nexus Between Social Work and the Environment. Australian Social Work, 6(3), 256-268. Doi: 10.1080/03124070802178275

Muxí, Z. (2015). Mujeres haciendo ciudades: Aprendiendo del pasado. Kult-ur, 2(3), 11-124. Disponible en: http://www.e-revistes.uji.es/index.php/kult-ur/article/view/1720/1535

Närhi, K. (2004). The Eco-social Approach in Social Work and the Challenges to the Expertise of Social Work. Jyvaskyla: University of Jyvaskyla. Recuperado de https://jyx.jyu.fi/dspace/bitstream/handle/123456789/13326/9513918343.pdf

Observatorio de Salud Pública de Santander (2005). Informe Especial. Emergencia de Invernal en Santander. Febrero de 2005. Recuperado de: http://web.observatorio.co/publicaciones/boletin_01-01-2005art00.pdf

Palacio, D. (2002). El parque nacional Utría, un Lugar-Red. Una propuesta de análisis socioambiental para la gestión de Áreas Protegidas. Territorios, 8, 39-61. Disponible en: https://revistas.urosario.edu.co/ index.php/territorios/article/viewFile/5683/3764

Palacio, D. (2015). Redes, Actores y Gobernanza desde un enfoque relacional. Colección hojas de ruta: guías para el estudio socio-ecológico de la alta montaña en Colombia, Libro 2. Bogotá: Instituto de Recursos Biológicos Alexander von Humboldt.

Palacio, D. (2017). El lugar-red y la acción ambiental. Pistas para una gobernanza reflexiva y situada. $R E-$ DES. Revista hispana para el análisis de redes sociales, 28(1), 73-91. Dispoonible en: http://revistes. uab.cat/redes/article/view/v28-n1-palacio/pdf-648-es

Palacio, D.C. (2001). Place-networks: a social - environmental Approach for conservation of Protected Areas. The case studies of Monte Tezio (Italy) and utria (Colombia). (Tesis doctoral. Swansea: University of Wales).

Pliego, F. (1994). Hacia una sociología de los desastres urbanos. México: Universidad Autónoma de México.

Pyles, L. (2015). Participation and other ethical considerations in participatory action research in post-earthquake rural Haiti. International Social Work, 58(5), 628-645. Doi: "https://doi. org/10.1177/0020872815581912"10.1177/0020872815581912

Pyles, L. (2017). Decolonising Disaster Social Work: Environmental Justice and Community Participation. The British Journal of Social Work, 47(3), 630-647. Doi: 10.1093/bjsw/bcw028.

Richmond, M. (2005 [1917]). Diagnóstico Social. Madrid: Siglo XXI.

Ríos, D. (2005). Planificación urbana privada y desastres de inundación: las urbanizaciones cerradas polderizadas en el municipio de Tigre, Economía, Sociedad y Territorio, 5(17), 63-83. Buenos Aires. Disponible en: http://www.redalyc.org/articulo.oa?id=11101704

Serna, C. (2011). La naturaleza social de los desastres asociados a inundaciones y deslizamientos en Medellín (1930-1990). Historia Crítica, 43, 198-223. Disponible en: http://www.redalyc.org/ pdf/811/81122475011.pdf

Thrift, N. (1997). On The Determination of Social Action in space and time. En: T. Barnes, y G. Derek, Reading Human Geography. The Poetics and Politics on Enquiry. (pp. 376-406). Londres.

Universidad Industrial de Santander (2000). Plan de Ordenamiento Territorial de San Juan Girón. 20002009. Bucaramanga: Centro de Estudios Regionales-UIS. 
Verde, C. (2013). Hull House: La ciencia al servicio de la reforma social. En: J. Addams, A. Lima y C. Verde, Hull House. El valor de un centro social (pp. 21-43). Madrid: Paraninfo y Consejo General de Trabajo Social.

Villasante, T. y Gutiérrez, M. (2006). Redes y Conjuntos de Acción: para aplicaciones estratégicas en los tiempos de la complejidad. REDES Revista hispana para el análisis de redes sociales, 11 (2). Disponible en: http://revistes.uab.cat/redes/article/view/v11-vilasante-martin-gutierrez/88

Wahlstrom, M. (2017). Social work and the Sendai Framework for Disaster Risk Reduction. European Journal of Social Work, 20(3), 333-336. Doi:10.1080/13691457.2017.1314936

Wasserman, S. y Faust, K. (1994). Social Network Analysis. Methods and Application. Structural Analysis in the social sciences, 8 . 